\title{
BMJ Open Public views towards community health and hospital-based outpatient services and their utilisation in Zhejiang, China: a mixed methods study
}

\author{
Dan Wu, ${ }^{1}$ Tai Pong Lam, ${ }^{1}$ Kwok Fai Lam, ${ }^{2}$ Xu Dong Zhou, ${ }^{3}$ Kai Sing Sun ${ }^{1}$
}

To cite: Wu D, Lam TP, Lam KF, et al. Public views towards community health and hospital-based outpatient services and their utilisation in Zhejiang, China: a mixed methods study. BMJ Open 2017;7:e017611. doi:10.1136/ bmjopen-2017-017611

- Prepublication history and additional material for this paper are available online. To view these files, please visit the journal online (http://dx.doi org/10.1136/bmjopen-2017017611).

Received 3 May 2017 Revised 20 September 2017 Accepted 16 October 2017

CrossMark

${ }^{1}$ Department of Family Medicine and Primary Care, Faculty of Medicine, The University of Hong Kong, Hong Kong, China

${ }^{2}$ Department of Statistics and Actuarial Science, Faculty of Science, The University of Hong Kong, Hong Kong, China

${ }^{3}$ Institute of Social Medicine, School of Public Health, Zhejiang University, Hangzhou, China

Correspondence to Professor Tai Pong Lam; tplam@hku.hk

\section{ABSTRACT}

Objective China is engaged in promoting community health services (CHS) nationwide. This study examines the public's views towards CHS and their utilisation of community-based and hospital-based outpatient services.

Design A mixed methods study using qualitative interviews and a cross-sectional survey.

Study setting and participants The study was conducted among the public between September 2014 and September 2015 in Zhejiang province, China. Six focus groups and 13 individuals were interviewed. The questionnaire was completed by 1248 respondents (response rate: 83\%).

Primary outcome measures Utilisation of community-based and hospital-based outpatient services. Results Functions of CHS perceived by the public included provision of minor illness management, coordination, drug dispensing, follow-up care and patient education. However, many also showed a distrust in primary care providers' (PCPs) competence for confirming the initial diagnosis and management plan. As coordinators, PCPs' integrity was challenged, and PCPs were thought to be potential 'tuo er' (cunning agents who tried to lead patients to some notorious hospitals to make money). Survey results showed that 800 (64.1\%) respondents visited hospital-based clinics and 688 (55.1\%) visited CHS at least once in the past year. Compared with the uninsured group, those covered by Urban Resident Medical Insurance (adjusted OR (AOR) $=1.95,95 \% \mathrm{Cl} 1.24$ to 3.07 ) and Urban Employee Medical Insurance (AOR=2.59, $95 \% \mathrm{Cl} 1.59$ to 4.24 ) were more likely to use hospital-based services. Respondents who had a chronic condition were more likely than their counterparts to use both hospitalbased services ( $\mathrm{AOR}=1.72,95 \% \mathrm{Cl} 1.18$ to 2.49 ) and $\mathrm{CHS}$ ( $\mathrm{AOR}=1.66,95 \% \mathrm{Cl} 1.19$ to 2.32). Income levels were positively associated with the likelihood of visiting hospital-based clinics (AOR=1.67, 95\% Cl 1.15 to 2.42) but negatively associated with the likelihood of using $\mathrm{CHS}$ ( $A 0 \mathrm{R}=0.68,95 \% \mathrm{Cl} 0.48$ to 0.96 ).

Conclusions Demand of hospital-based outpatient services is much higher than the community-based outpatient services. Policy reformers need to take further actions to address the public distrust in PCPs to facilitate their gatekeeping role.

\section{INTRODUCTION}

China has a three-tier healthcare system in which secondary and tertiary hospitals are supposed to provide specialised care, while
Strengths and limitations of this study

- Our qualitative part provided the in-depth views of service users towards the community health services.

- The quantitative data triangulated the qualitative findings using a follow-up cross-sectional survey approach.

- The study mapped out the public's utilisation of both community-based and hospital-based outpatient services in a free market style healthcare system in the urban setting.

- The study was conducted in a pioneer city implementing the national healthcare reforms and provided references to other areas of China with similar situations.

- The quantitative part of the study was a selfadministered questionnaire survey that might introduce recall bias regarding past experiences.

community health facilities provide primary care and preventive services. However, patients embrace the freedom to choose any level of care facility and direct access to specialist care without referrals by primary care doctors. The country has been engaged in a massive healthcare reform since 2009. Strengthening primary care system and diverting patients with common diseases from hospital-based care to community-based care is one of the top five priorities of the reform. ${ }^{1}$ Nevertheless, the reform would not be successful if the voices of the service users are ignored. Previous research focused on patients' satisfaction in health services, including primary care. $^{2-5}$ Other studies attempted to explore patients' willingness to choose community health services (CHS) for first-contact care, of which percentages ranged between $62 \%$ and $70 \%,{ }^{6-8}$ but our recent study found $70 \%$ of the respondents sampled from the general public preferred hospital-based services for first-contact care. ${ }^{9}$ Associated factors included patient's age, sex, education level, insurance 
status, awareness of CHS and self-perceived severity of diseases. ${ }^{781011}$ Better accessibility and affordability were considered as strengths of CHS. ${ }^{12} \mathrm{~A}$ few studies reported patients' concerns about the competence of primary care providers (PCPs) and quality of diagnostic equipment. ${ }^{9}{ }^{13-15}$ The general public's in-depth views towards CHS are lacking.

This study is part of a larger project examining how to better promote primary care in China using a combined qualitative and quantitative approach. The first aim of this article was to examine the general public's views towards the roles of CHS through the qualitative study. In China, PCPs refer to any type of medical practitioners, regardless of educational attainment, providing primary care in the community. PCPs include general practitioners (who have 3-year or 5-year medical education and are specialised in general practice through general practice training programmes), barefoot doctors (3-6 months of basic medical training) and other medical practitioners providing primary care in the community setting. Because the service users are free to choose between facilities, it is possible that they seek medical help from multiple resources. The second aim was to investigate through a questionnaire survey their utilisation of community-based and hospital-based outpatient services in the year prior to the study, with a focus on the former. Since China's previous primary care system established between 1960s and 1980s has been a successful model for lower income and middle-income countries, ${ }^{16}$ the Chinese experiences are relevant to other countries.

\section{METHODS}

The study method was described elsewhere. ${ }^{9}$ Data were collected from the general public living in the eight districts of central Hangzhou, with a population of 6.95 million, in Zhejiang province.

\section{Qualitative approach}

Sampling and data collection

We adopted both individual and focus group interviews to triangulate data. We purposefully sampled participants with diverse backgrounds for interviews. For individual interviews, we recruited participants from different age groups, with different education levels, income levels and whether diagnosed as having chronic conditions or not. Group interviews were mainly arranged based on age groups with flexibility allowed. We identified a local researcher who had strong connections with local health authorities. We approached participants with the assistance of community workers.

Topic guides were developed for semistructured interviews. This article focused on the participants' views of the roles of CHS and PCPs. Interviews were conducted between September 2014 and April 2015. Most interviews lasted around $60 \mathrm{~min}$ and were conducted in a quiet room in the community. Written consent was sought before the interview. Anonymity and confidentiality were stressed.

\section{Data analysis}

Interviews were audio-recorded and transcribed verbatim. One of the authors checked the accuracy of the transcripts. Data were analysed using a thematic approach with the assistance of NVivo V.10. Interview transcripts were double coded by two researchers to check for consistency and accuracy. Discrepancies were then discussed until consensus was reached. Afterwards, we organised and categorised codes into different themes/subthemes, and further relevant questions were explored in subsequent interviews. We continued data collection until thematic saturation was reached. To ensure data validity, some key findings were triangulated by a follow-up survey. Quotes were translated from Chinese to English for write-up.

\section{Quantitative approach}

\section{Sampling and data collection}

A cross-sectional survey was conducted from July to September 2015. Multistage stratified random sampling was adopted. First, we stratified the eight districts to low, middle and high-income regions. One district from each stratum was then randomly selected. Second, two street districts were randomly selected from each district. Then, four communities/villages were randomly selected from each sampled street district, making it a total of 24 survey sites. Lastly, individuals were randomly selected and approached with the assistance of the community workers, and we recruited 45-50 respondents from each site. Anonymity was stressed, and confidentiality was strictly protected.

\section{Questionnaire}

The complete questionnaire has 64 items. We asked the participants about their social demographic characteristics, insurance scheme (New Cooperative Medical Insurance (NCMI), Urban Resident Medical Insurance (URMI), Urban Employee Medical Insurance (UEMI) and commercial insurance), factors they consider for finding sources of care, preferred source of care, use of outpatient services in the last year, expectations on medical professionals in the clinical setting and views of CHS and PCPs. NCMI covered rural population, URMI covered people who were under 18 years old, the unemployed or poor, the disabled and the elderly population, while UEMI covered employees in urban areas. In this article, we reported the public's utilisation of CHS and hospital-based clinics in the last year and the correlates. Experiences and views of community-based services were asked using 4-point Likert scales ( $1=$ completely disagree; 2=somewhat disagree; $3=$ somewhat agree; $4=$ completely agree). The questionnaire was piloted with 30 respondents to test its face and content validity. The questionnaire aimed at investigating the attitudes and behaviours of the respondents but did not serve as a composite measure of a certain domain. Hence, it has not been validated against outcomes formally other than the face and content validity. Completing the questionnaire took 10-15 min. Amendments were made based on feedback. 


\section{Statistical analysis}

The data were analysed using IBM SPSS V.23. We had dichotomised the responses by grouping 'completely agree and somewhat agree' as positive response and 'completely disagree and somewhat disagree' as negative response. Number of visits to CHS and hospitals were categorised as 'none' and 'at least once' for further multiple logistic regression analysis. Descriptive analysis was carried out and Pearson $\chi^{2}$ tests were conducted to examine the differences in the views of PCPs by age and number of visits to CHS in the last year. We ran simple logistic regression and multiple logistic regression analyses to determine the factors (ie, sex, age, income, health insurance status and chronic conditions) for the use of health services and public opinions of PCPs. A p Value $<0.05$ is considered statistically significant.

\section{RESULTS}

\section{Qualitative results}

Six focus group interviews were held. Each group comprised six to eight participants. Meanwhile, 13 semistructured individual interviews were conducted. Among the participants, $44.4 \%$ were males. The mean age of all participants was $52.6(\mathrm{SD}=17.0)$, with the age ranged from 24 to 87 . Views towards the roles of CHS and perceptions of PCPs were revealed.

\section{Roles of CHS}

Many participants perceived that CHS were like drug dispensers rather than a medical care provider (online supplementary appendix 1, quotes 1 and 2). CHS were regarded as a source for dealing with minor illnesses, such as common colds and skin abrasions (online supplementary appendix 1 , quote 3 ). Nevertheless, the public's perception of minor and urgent/serious conditions might be largely different from the professionals' views and varied across different participants. For example, fevers and abdominal discomfort were considered by some as conditions that need hospital specialist consultations to confirm the causes (online supplementary appendix 1, quotes 4 and 5). When the interviewer asked 'what proportion of your health problems can be dealt with by primary care providers', the responses widely ranged from $20 \%$ to $90 \%$.

Elderly patients with chronic diseases can be well managed in CHS (online supplementary appendix 1, quote 6). Some participants described it as a source for follow-up care for chronic diseases but not for confirming the initial diagnosis and management plan even for diabetes and hypertension (online supplementary appendix 1, quotes 7 and 8). A key role played by CHS was to provide general and public health education to the general public, as explained by a participant (online suppementary appendix 1, quote 9). The function of triaging or making referrals by CHS was also discussed by some participants. CHS could inform patients to make better decisions about which specialty to see or where to see a specialist (online supplementary appendix 1, quotes
10 and 11). Such a status quo was deemed by them as a step forward compared with the past (online supplementary appendix 1, quote 11). However, the lack of trust in PCPs' integrity might pose a challenge for this function. For the question 'what would you think about a PCP making an appointment with hospital specialists for you, as a coordinator?', an interviewee responded that he would doubt if the PCP was a 'tuo er' (online supplementary appendix 1 , quote 12).

\section{Perceptions towards PCPs}

Many participants frankly expressed PCPs were like barefoot doctors with minimal formal medical education (online supplementary appendix 2 , quote 1 ). The local dialects used were 'san jiao mao' (a cat with three $\operatorname{legs}^{\mathrm{ii}}$ ) and 'wan jin you' (Tiger Balm ${ }^{\mathrm{iii}}$ ), meaning that PCPs saw any diseases yet were not good at any kind of them (online supplementary appendix 2, quotes 2 and $3)$. In the doctor-patient relationship, patients sometimes appeared to play a dominant role and requested treatments (online supplementary appendix 2, quotes 4 and 5). Using 'san jiao mao' or 'wan jin you' as a metaphor to describe PCPs demonstrated a strong public distrust in the level of competence of PCPs (online supplementary appendix 2, quotes 6 and 7). One major cause was the participants' belief that PCPs were inadequately educated and trained (online supplementary appendix 2, quote 7 ).

\section{Survey results}

A total of 1248 respondents completed the questionnaire. The overall response rate was $83 \%$. Nearly half $(49.4 \%)$ of the respondents were female. There were 132 (10.6\%) aged 60 years or above. Five hundred and four $(40.4 \%)$ respondents obtained postsecondary or higher education and $393(31.5 \%)$ reported a monthly household income of 10000 yuan or above. Nearly one-fourth $(325,26.0 \%)$ were covered by NCMI, $388(31.1 \%)$ were covered by URMI and $272(21.8 \%)$ were covered by UEMI. Over one-fifth $(265,21.2 \%)$ had a chronic condition. Details of their demographic characteristics have been reported elsewhere. ${ }^{9}$ The majority $(800,64.1 \%, 95 \%$ CI $61.4 \%$ to $66.7 \%$ ) visited hospital-based clinics in the past year. Less $(688,55.1 \%, 95 \%$ CI $52.4 \%$ to $57.9 \%)$ visited community-based clinics, $507(74.2 \%)$ of whom also visited hospital clinics in the past year. Detailed distribution of the number of visits to the two types of clinic are shown in table 1 .

\footnotetext{
i 'Tuo er' is a Beijing dialect which means a cunning agent who pretends to be a client and tries to deceive the real customers and lead them to buy their products. It exists in many industries. In health industry, it means an agent who tries to lead patients to some notorious hospitals to make money.

ii A popular idiom in Yangtze River Delta. It means jack of all trades and master of none

iii A Chinese relieving ointment which can be applied for many conditions such as skin itchiness, headache, rheumatic pains, and diarrhoea etc., leading to mild relief but not curing the diseases.
} 
Table 1 Visits to community-based and hospital-based services in the year prior to the survey $(n=1248)$

\begin{tabular}{lllllll}
\hline Number of visits (times) & $\mathbf{0}(\%)$ & $\mathbf{1 - 3}(\%)$ & $\mathbf{4 - 6}(\%)$ & $\mathbf{7 - 1 0 ~ ( \% )}$ & $\mathbf{> 1 0}(\%)$ & Missing \\
\hline Community health facilities & $485(38.9)$ & $455(36.5)$ & $125(10.0)$ & $42(3.4)$ & $66(5.3)$ & $75(6.0)$ \\
Hospital outpatient clinics & $376(30.1)$ & $557(44.6)$ & $154(12.3)$ & $41(3.3)$ & $48(3.8)$ & $72(5.8)$ \\
\hline
\end{tabular}

Table 2 shows the associations between individual characteristics and the outpatient services utilisation based on the simple and multiple logistic regression analyses. Compared with the uninsured group, those covered by URMI (adjusted OR (AOR) $=1.95,95 \%$ CI 1.24 to 3.07 ) and UEMI (AOR=2.59, 95\% CI 1.59 to 4.24 ) were significantly more likely to use hospital-based services. Those who reported the highest household income (AOR $=1.67$, $95 \%$ CI 1.15 to 2.42 ) and a diagnosis of chronic illnesses (AOR $=1.72,95 \%$ CI 1.18 to 2.49 ) were more likely to use hospital-based services. In relation to community-based services, reporting a diagnosis of chronic illnesses was significantly associated with the utilisation $(\mathrm{AOR}=1.66$, $95 \%$ CI 1.19 to 2.32 ), whereas the likelihood significantly decreased with income levels (AOR $=0.68$, 95\% CI 0.48 to 0.96$)$.
Among the 688 CHS users, 464 (67.4\%) offered comments about their experiences of using CHS (table 3). Over half of them (54.3\%) reported that they had a regular PCP, with significantly more respondents aged 60 years or above reporting this than the other two age groups $(p=0.020)$. Main reasons for visiting a community-based practitioner were refilling medications $(56.0 \%)$ and seeking professional advice $(65.0 \%)$. More from the elderly $(84.7 \%)$ and middle-aged $(64.2 \%)$ groups than the youngest group $(45.0 \%)$ went to CHS to buy or refill medications $(\mathrm{p}<0.001)$. Almost $85 \%$ said that PCPs were polite to them. Up to $87.3 \%$ reported that PCPs listened to patients carefully. Nearly $81 \%$ reported that PCPs explained the disease, management plan and drug adverse effects patiently. Two-thirds said that PCPs cared about their mental health $(66 \%)$, were empathetic

Table 2 Correlates of use of hospital-based and community-based outpatient services

\begin{tabular}{|c|c|c|c|c|}
\hline & \multicolumn{2}{|c|}{$\begin{array}{l}\text { OR and adjusted OR of visiting a hospital } \\
\text { specialist last year at least once }\end{array}$} & \multicolumn{2}{|c|}{$\begin{array}{l}\text { OR and adjusted OR of visiting a community } \\
\text { based clinic last year at least once }\end{array}$} \\
\hline & OR (95\% CI) & AOR (95\% CI) & OR $(95 \% \mathrm{Cl})$ & AOR $(95 \% \mathrm{Cl})$ \\
\hline Male & 1 & 1 & 1 & 1 \\
\hline Female & 1.19 (0.93 to 1.53$)$ & 1.16 (0.88 to 1.52$)$ & $1.29(1.02 \text { to } 1.63)^{*}$ & 1.26 (0.98 to 1.62$)$ \\
\hline $40-59$ & 1.20 (0.89 to 1.62$)$ & $1.11(0.79$ to 1.55$)$ & 1.09 (0.82 to 1.44$)$ & 0.94 (0.69 to 1.28$)$ \\
\hline 60 or above & $1.16(0.77$ to 1.74$)$ & $0.70(0.43$ to 1.14$)$ & 1.48 (0.99 to 2.20$)$ & $1.16(0.73$ to 1.84$)$ \\
\hline \multicolumn{5}{|l|}{ Insurance status } \\
\hline Uninsured & 1 & 1 & 1 & 1 \\
\hline Commercial insurance & 1.07 (0.55 to 2.08) & 1.03 (0.51 to 2.08$)$ & $0.89(0.46$ to 1.71$)$ & 0.97 (0.49 to 1.94$)$ \\
\hline \multicolumn{5}{|l|}{ Income } \\
\hline$<4000$ & 1 & 1 & 1 & 1 \\
\hline 4000-9999 & 1.19 (0.88 to 1.62$)$ & 1.09 (0.78 to 1.52$)$ & 0.78 (0.58 to 1.06$)$ & 0.76 (0.55 to 1.05$)$ \\
\hline$>10000$ & $1.85(1.33 \text { to } 2.58)^{\star \star \star}$ & $1.67(1.15 \text { to } 2.42)^{* \star}$ & $0.63(0.46 \text { to } 0.86)^{\star *}$ & $0.68(0.48$ to 0.96$){ }^{*}$ \\
\hline \multicolumn{5}{|l|}{ Chronic illness diagnosis } \\
\hline No & 1 & 1 & 1 & 1 \\
\hline Yes & $1.70(1.23 \text { to } 2.34)^{\star \star}$ & $1.72(1.18$ to 2.49$){ }^{* \star}$ & $1.68(1.25 \text { to } 2.25)^{\star \star}$ & $1.66(1.19$ to 2.32$)$ ** \\
\hline
\end{tabular}

Model fit descriptions for multiple logistic regression analysis: $p$ Values of Hosmer and Lemeshow test, respectively, for the two dependent variables are 0.456 and 0.454 , indicating that the models fit the data well.

${ }^{*} \mathrm{p}<0.05,{ }^{* *} \mathrm{p}<0.01,{ }^{* *} \mathrm{p}<0.001$.

NCMI, New Cooperative Medical Insurance; UEMI, Urban Employee Medical Insurance; URMI, Urban Resident Medical Insurance. 
Table 3 Community health service users' experiences and their views of PCPs by age $(n=464)$

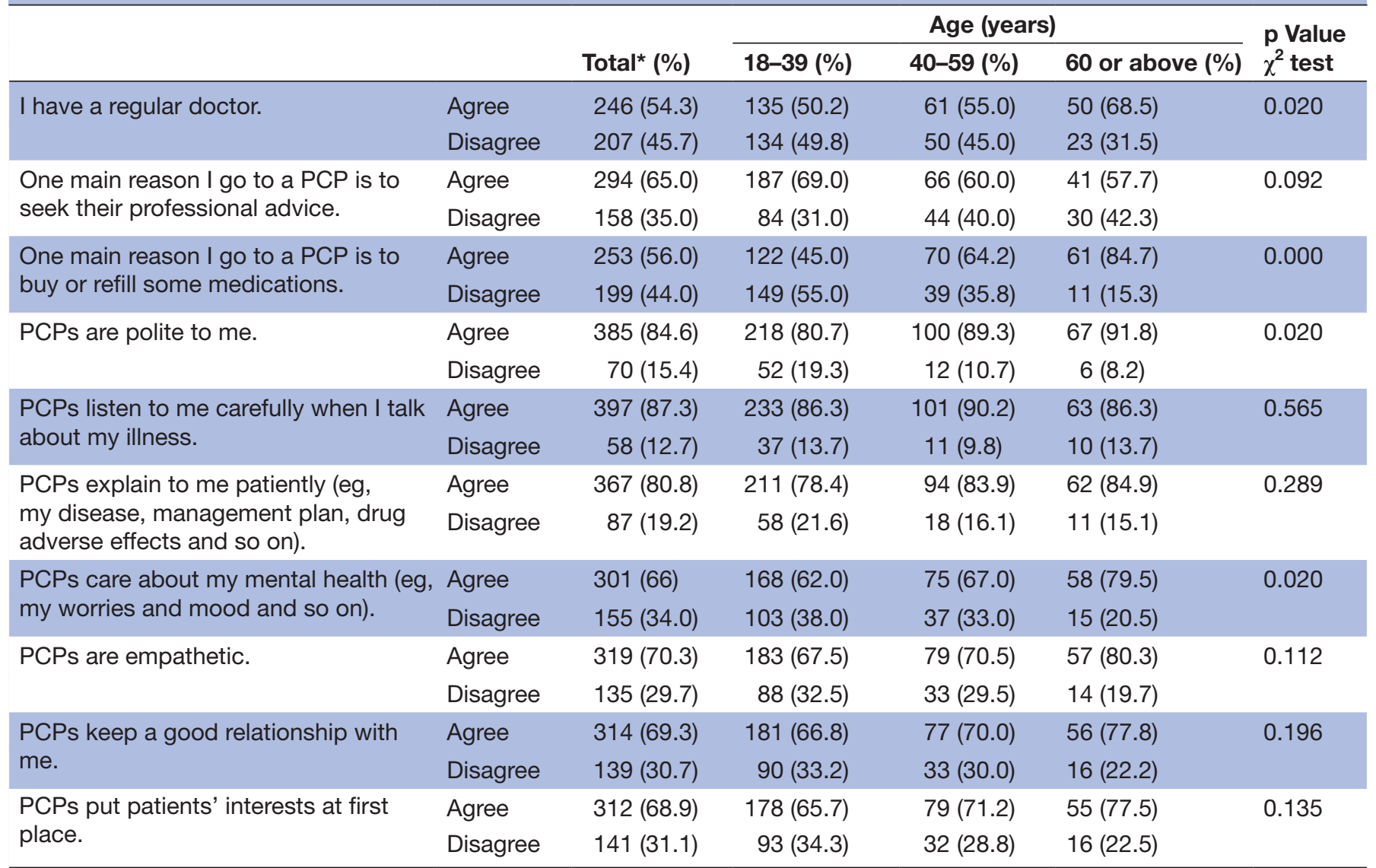

*The numbers do not add up because of missing ages in some of the responses.

$\mathrm{PCP}$, primary care provider.

$(70.3 \%)$, tried to keep a good relationship with the patient $(69.3 \%)$ and put patients' interests at the first place $(68.9 \%)$.

There were differences in their views by number of visits to CHS (table 4). The percentages of reporting PCPs caring about their mental health status $(\mathrm{p}=0.008)$, and putting patients' interests at first place $(\mathrm{p}=0.038)$ increased with the number of visits to CHS. Further, the percentage of reporting a purpose for refilling medications at CHS was higher among the frequent visitors of CHS, compared with those who visited less frequently $(p=0.012)$. After controlling for sex, insurance status and income, multiple logistic regression analysis revealed that, compared with the age group 18-39 years, those aged over 60 years were significantly more likely to have reported the purpose of refilling medication at $\mathrm{CHS}$ (AOR=5.59, 95\% CI 2.62 to 11.92) and that PCPs cared about their mental health (AOR=2.04, 95\% CI 1.00 to 4.13). Visiting over 10 times in the last year had a significant positive correlation with reporting PCPs caring about their mental health (AOR=3.42, 95\% CI 1.33 to 8.81), keeping a good relationship (AOR=3.08, 95\% CI 1.23 to 7.69) and putting patients' interests as the priority $(\mathrm{AOR}=2.97$, 95\% CI 1.19 to 7.39 ), compared with those who paid one to three visits. However, only $43(9.3 \%, 95 \%$ CI $7.0 \%$ to $12.3 \%$ ) reported that over $75 \%$ of their medical problems were managed by PCPs and $60.2 \%$ reported that $50 \%$ or less of their medical problems were managed by PCPs when they felt ill and sought help from a doctor.

\section{DISCUSSION}

Demand of hospital-based outpatient services is much higher than the community-based outpatient services indicating a wastage of health resources. In a healthcare system that allows a large degree of freedom to choose any health institution for healthcare, patients choose hospitals that they think can provide better care. A minority thought that PCPs managed over $75 \%$ of their medical problems, indicating a widespread lack of confidence in PCPs' competence. Many participants perceived PCPs as inferior in managing diseases of specific organ systems than specialists. Their perceptions might also be affected by historical factors. PCPs were deemed not different from barefoot doctors who, although with inadequate training, met basic healthcare needs of the rural population from 1960s to 1980s but less so at the present time. The bad experiences, such as not getting satisfactory explanations from a PCP, reinforced such perceptions.

Inadequate formal medical education and training for PCPs is an underlying problem. In 2013, around $76 \%$ of PCPs in urban community health facilities obtained 
Table 4 CHS users' experiences and their views of PCPs by number of visits to $\mathrm{CHS}(n=464)$

\begin{tabular}{|c|c|c|c|c|c|c|c|}
\hline & & & & Number & visits to $C$ & IS (times) & \\
\hline & & Total $^{*}(\%)$ & $1-3(\%)$ & 4-6 (\%) & 7-10 (\%) & Over $10(\%)$ & p Value \\
\hline I have a regular doctor. & Agree & $244(54.8)$ & $134(50.2)$ & $41(56.9)$ & $17(60.7)$ & $32(69.6)$ & 0.106 \\
\hline & Disagree & $201(45.2)$ & $133(49.8)$ & $31(43.1)$ & $11(39.3)$ & $14(30.4)$ & \\
\hline One main reason I go to a PCP & Agree & $292(65.8)$ & $174(65.7)$ & 52 (73.2) & $19(67.9)$ & $26(54.2)$ & 0.321 \\
\hline $\begin{array}{l}\text { is to seek their professional } \\
\text { advice. }\end{array}$ & Disagree & $152(34.2)$ & $91(34.3)$ & $19(26.8)$ & $9(32.1)$ & $22(45.8)$ & \\
\hline One main reason I go to a & Agree & 248 (55.9) & $135(50.9)$ & $44(62.0)$ & $16(57.1)$ & $37(77.1)$ & 0.012 \\
\hline $\begin{array}{l}\text { PCP is to buy or refill some } \\
\text { medications. }\end{array}$ & Disagree & $196(44.1)$ & $130(49.1)$ & $27(38.0)$ & $12(42.9)$ & $11(22.9)$ & \\
\hline PCPs are polite to me. & Agree & $379(84.8)$ & $221(82.5)$ & $62(84.9)$ & $25(89.3)$ & 45 (95.7) & 0.202 \\
\hline & Disagree & $68(15.2)$ & $47(17.5)$ & $11(15.1)$ & $3(10.7)$ & $2(4.3)$ & \\
\hline PCPs listen to me carefully when & Agree & $389(87.0)$ & $229(85.8)$ & $67(91.8)$ & $23(82.1)$ & $44(91.7)$ & 0.460 \\
\hline I talk about my illness. & Disagree & $58(13.0)$ & $38(14.2)$ & $6(8.2)$ & $5(17.9)$ & $4(8.3)$ & \\
\hline PCPs explain to me patiently & Agree & $360(80.7)$ & 209 (78.0) & $61(83.6)$ & $21(77.8)$ & $41(87.2)$ & 0.294 \\
\hline $\begin{array}{l}\text { (eg, my disease, management } \\
\text { plan, drug adverse effects and } \\
\text { so on). }\end{array}$ & Disagree & $86(19.3)$ & $59(22.0)$ & $12(16.4)$ & $6(22.2)$ & $6(12.8)$ & \\
\hline PCPs care about my mental & Agree & $292(65.2)$ & $164(61.2)$ & $45(61.6)$ & $18(64.3)$ & 42 (87.5) & 0.008 \\
\hline & Disagree & $156(34.8)$ & $104(38.8)$ & $28(38.4)$ & $10(35.7)$ & $6(12.5)$ & \\
\hline PCPs are empathetic (eg, my & Agree & $314(70.4)$ & $187(69.8)$ & $48(66.7)$ & $20(71.4)$ & $39(83.0)$ & 0.327 \\
\hline w & Disagree & $132(29.6)$ & $81(30.2)$ & $24(33.3)$ & $8(28.6)$ & $8(17.0)$ & \\
\hline a good relationship & Agree & $309(69.3)$ & $174(65.2)$ & $52(71.2)$ & $20(71.4)$ & $40(85.1)$ & 0.085 \\
\hline & Disagree & $137(30.7)$ & 93 (34.8) & $21(28.8)$ & $8(28.6)$ & $7(14.9)$ & \\
\hline PCPs put patients' interests at & Agree & $310(69.5)$ & 175 (65.5) & $54(74.0)$ & 22 (78.6) & $40(85.1)$ & 0.038 \\
\hline & Disagree & $136(30.5)$ & $92(34.5)$ & $19(26.0)$ & $6(21.4)$ & 7 (14.9) & \\
\hline
\end{tabular}

${ }^{*}$ The numbers do not add up because of missing values in some of the responses.

$\mathrm{CHS}$, community health services; PCP, primary care provider.

three or more years of formal medical education and this compared with $90 \%$ of hospital specialists. ${ }^{17}$ Doctors showing clinical competence is critical to gaining patients' trust, which lays a foundation for a healthy doctor-patient relationship. ${ }^{18}$ Improving PCPs' competence through bettering the primary care education system is a top priority for building a strong primary care system and the subsequent success of health reforms in China.

Besides, those with higher household income and urban residents covered by URMI and UEMI were more likely to use hospital-based services. People may be inclined to put their preference into practice when they can afford the service. The NCMI appears to have no impact on their utilisation of hospital-based clinic. This might be due to the lower reimbursement rate for self-referred patients at hospitals and poorer out-of-pocket payment capacity by rural-to-urban migrants. Better-off patients with chronic diseases need long-term care that generally increases the chances for visiting doctors in both hospitals for specialist care and CHS for follow-up care or refilling medications.

One perceived function of CHS in the public's eyes is minor illness management. However, the public's understanding may be greatly different from the professionals' views. Common conditions, such as fevers and abdominal discomfort, most of which are minor, are considered by some patients as conditions that need hospital specialists' consultations or diagnostic tests to confirm the causes and management plans. CHS is also considered a referral agency that could advise patients of a preferred hospital specialist. This is a positive trend because, if the public are willing to accept CHS as a referral body, to some extent, CHS can function as a gatekeeper and coordinator. Helping patients make better informed decisions of seeing appropriate specialists has the potential to reduce unnecessary care considerably.

Other roles of CHS, which are well accepted by the public, include drug dispensing, managing patients with chronic diseases and patient education. Although the service users lack confidence in CHS as a provider of first-contact care, ${ }^{9}$ providing aforementioned quality services to patients in need can gradually build up a rapport with the patients and regain trust. As our survey identifies, more contact with PCPs may contribute to positive public opinions towards PCPs regardless of the reasons for encounters. Strengthening these functions are likely to be a good starting point for CHS to attract and keep patients in primary care. Nevertheless, frequent report for refilling medications may not be clinically 
indicated and whether there are unnecessary visits due to refilling medications are worth further examination. Also, 'Tuo er' mentioned by one participant indicates that a deep distrust in PCPs' integrity in acting as a coordinator may exist, which may be worth further actions.

This study has some strengths and limitations. The data were collected in a developed urban city where hospitals are easily accessible to the general public. The study findings only relate to Zhejiang province and may not be generalisable to less developed, rural areas. However, as one of the earliest health reform pilot cities, Hangzhou has developed a highly accessible and affordable primary care system. The findings provide a snapshot of the public's healthcare utilisation patterns in the context of an intensive healthcare reform. Then a self-administered questionnaire might introduce recall bias regarding past experiences. We designed the experience questions using ranges to make it easier to respond. The items on their views and attitudes are subjective in nature, and concerns about recall bias are inconsequential. Furthermore, our qualitative study enables us to acquire an in-depth understanding of their thoughts.

\section{CONCLUSION}

The study aimed to investigate the public's views towards CHS and their healthcare utilisation experiences. Demand of hospital-based outpatient services is much higher than the community-based outpatient services. The public's perceptions of PCPs reflect a deep distrust in their competence. The distrust places a huge obstacle to CHS assuming the role of first-contact care provider. Nevertheless, other functions like making referrals, providing follow-up care and health education are well accepted and can be a good starting point for CHS to earn public trust. The gatekeeping role shall be gradually adopted by CHS after a strong primary care workforce is established by a primary care-oriented education system.

Contributors This research formed part of DW's PhD project. DW, TPL and XDZ designed the study and the questionnaire. DW and XDZ coordinated fieldwork and collected data. DW, KSS and KFL performed the data analysis. DW, KSS and TPL interpreted the analysis. DW, KSS and TPL drafted the manuscript. All authors reviewed, revised and approved the final manuscript.

Funding This work was supported by Committee on Research and Conference Grants of The University of Hong Kong (Grant Number 201409176026). The funding body has not involved in study design, data collection, data analysis or the writing of the article.

\section{Competing interests None declared.}

Ethics approval Ethical approvals were obtained from the review boards of The University of Hong Kong/Hospital Authority Hong Kong West Cluster (UW14-567) and Zhejiang University (ZGL201410H), respectively.

Provenance and peer review Not commissioned; externally peer reviewed.
Data sharing statement Our study also collected data from medical professionals. These data are not published and are available to the corresponding author and the research team only.

Open Access This is an Open Access article distributed in accordance with the Creative Commons Attribution Non Commercial (CC BY-NC 4.0) license, which permits others to distribute, remix, adapt, build upon this work non-commercially, and license their derivative works on different terms, provided the original work is properly cited and the use is non-commercial. See: http://creativecommons.org/ licenses/by-nc/4.0/

(C) Article author(s) (or their employer(s) unless otherwise stated in the text of the article) 2017. All rights reserved. No commercial use is permitted unless otherwise expressly granted.

\section{REFERENCES}

1. Chen Z. Launch of the health-care reform plan in China. Lancet 2009;373:1322-4.

2. Li J, Wang P, Kong $X$, et al. Patient satisfaction between primary care providers and hospitals: a cross-sectional survey in Jilin province, China. Int J Qual Health Care 2016;28:346-54.

3. Lou Y, Zheng ZQ. Community residents' satisfaction with communith health services in Fuzhou (in Chinese). Chin Community Doct 2011;13:349-50.

4. Tang $\mathrm{L}$. The Chinese community patients life satisfaction, assessment of community medical service, and trust in community health delivery system. Health Qual Life Outcomes 2013;11:18.

5. Ren Y, Zhang TH, Shane T, et al. An investigation of patients' experience on using community health services in Beijing (in Chinese). Chin Gen Pract 2007;10:1764-7.

6. Zhang P, Zhao L, Liang J, et al. Societal determination of usefulness and utilization wishes of community health services: a population-based survey in Wuhan city, China. Health Policy Plan 2015;30:1243-50.

7. Gan Y, Li W, Cao S, et al. Patients' willingness on community health centers as gatekeepers and associated factors in Shenzhen, China: a cross-sectional Study. Medicine 2016;95:e3261.

8. Tang C, Luo Z, Fang P, et al. Do patients choose community health services (CHS) for first treatment in China? Results from a community health survey in urban areas. $J$ Community Health 2013;38:864-72.

9. Wu D, Lam TP, Lam KF, et al. Health reforms in china: the public's choices for first-contact care in urban areas. Fam Pract 2017;34:194-200.

10. Lian YS, Fan Q, Chen YN. Utilization situation and satisfaction of community health services in Nanjing City (in Chinese). Chin Gen Pract 2010;13:2086-8.

11. Zhou XJ, Gao LC, Xi QH. Motivation and satisfaction of community health service users (in Chinese). Chin Gen Pract 2009;12:2003-5.

12. Zhao WM, Cui MX, Li M. Yinchuan community health service present situation and satisfaction degree of the residents (in Chinese). Chin Gen Pract 2008;11:434-6.

13. McCollum R, Chen L, ChenXiang T, et al. Experiences with primary healthcare in Fuzhou, urban China, in the context of health sector reform: a mixed methods study. Int $J$ Health Plann Manage 2014;29:e107-26.

14. Yang Y, Yang D. Community health service centers in China, not always trusted by the populations they serve? China Econ Rev 2009;20:620-4.

15. Wang H, Gusmano MK, Cao Q. An evaluation of the policy on community health organizations in China: will the priority of new healthcare reform in China be a success? Health Policy 2011;99:37-43

16. Weiyuan $\mathrm{C}$. China's village doctors take great strides. Bull World Health Organ 2008;86:914-5.

17. Wu D, Lam TP. At a crossroads: family medicine education in China. Acad Med 2017;92:185-91.

18. Thom DH. Stanford Trust Study Physicians. Physician behaviors that predict patient trust. J Fam Pract 2001;50:323-8. 